\title{
Two-dimensional depth-resolved Mueller matrix of biological tissue measured with double-beam polarization-sensitive optical coherence tomography
}

\author{
Shuliang Jiao and Lihong V. Wang \\ Optical Imaging Laboratory, Biomedical Engineering Program, Texas A\&M University, 3120 TAMU, College Station, Texas 77843-3120
}

Received August 10, 2001

\begin{abstract}
A double-beam polarization-sensitive system based on optical coherence tomography was built to measure the Mueller matrix of scattering biological tissue with high spatial resolution. The Jones matrix of a sample can be determined with a single scan and subsequently converted into an equivalent nondepolarizing Mueller matrix. As a result, the system can be used to measure the Mueller matrix of an unstable sample, such as soft tissue. The polarization parameters of a porcine tendon, including magnitude and orientation of birefringence and diattenuation, were extracted by decomposition of the measured Mueller matrix. (C) 2002 Optical Society of America
\end{abstract}

OCIS codes: $120.2130,170.4500,260.5430,290.7050$.

Polarization-sensitive optical coherence tomography (OCT) is emerging as a very attractive branch of OCT. Polarization-sensitive OCT can reveal important information about biological tissue, such as birefringence, which is unavailable in conventional OCT. ${ }^{1-3}$ Birefringence is related to various biological components, such as collagen, muscle fibers, keratin, retina, and glucose; consequently, polarization can potentially provide new contrast mechanisms. The best way to ascertain the optical polarization properties of a sample is to measure its Mueller matrix. The combination of polarimetry and OCT makes it possible to acquire the Mueller matrix of a sample with OCT resolution. ${ }^{4,5}$ Yao and Wang ${ }^{4}$ first reported the measurement of Mueller-matrix images of biological tissues with OCT. Jiao et al. ${ }^{5}$ further demonstrated that the degree of polarization of the backscattered light measured with OCT remains unity throughout the detection range-indicating that the measured Mueller matrix is nondepolarizing-because OCT detects the coherent part of the electric field of the backscattered light regardless of whether it has been multiply scattered. This conclusion allows the use of the Jones matrix in OCT. The relatively timeconsuming process of these previous measurements of Mueller matrices limited the technique to use with stable samples such as bones. To measure unstable samples such as soft tissues, we have developed a system that can determine the Mueller matrix with a single depth scan (A scan). In other words, this system can acquire the Mueller matrix as quickly as its conventional OCT counterpart acquires a regular image.

In this Letter we report on our latest OCT system, which can acquire the depth-resolved Jones matrix of a sample with a single A scan. The Jones matrix, $\mathbf{J}$, is then transformed into an equivalent nondepolarizing Mueller matrix by ${ }^{6}$

$$
\mathbf{M}=\mathbf{U}\left(\mathbf{J} \otimes \mathbf{J}^{*}\right) \mathbf{U}^{-1},
$$

where $\otimes$ represents the Kronecker tensor product and $\mathbf{U}$ is the $4 \times 4$ Jones-Mueller transformation matrix
$[1,0,0,1 ; 1,0,0,-1 ; 0,1,1,0 ; 0, i,-i, 0]$, which is written row by row. The Jones matrix has four complex elements, in which seven real parameters are independent. At least two independent incident polarization states, which are not necessarily orthogonal to each other, are needed to determine completely a Jones matrix.

The schematic of the experimental setup is shown in Fig. 1. Two superluminescent diodes (central wavelength $\lambda=850 \mathrm{~nm}$, FWHM bandwidth $\Delta \lambda=26 \mathrm{~nm}$ ) are employed as low-coherence light sources and are amplitude modulated at 3 and $3.5 \mathrm{kHz}$ by variation of the injection current. The two source beams are merged by a polarizing beam splitter, filtered by a spatial filter assembly, and then split into the reference and sample arms by a nonpolarizing beam splitter. The sample beam passes through a quarter-wave plate

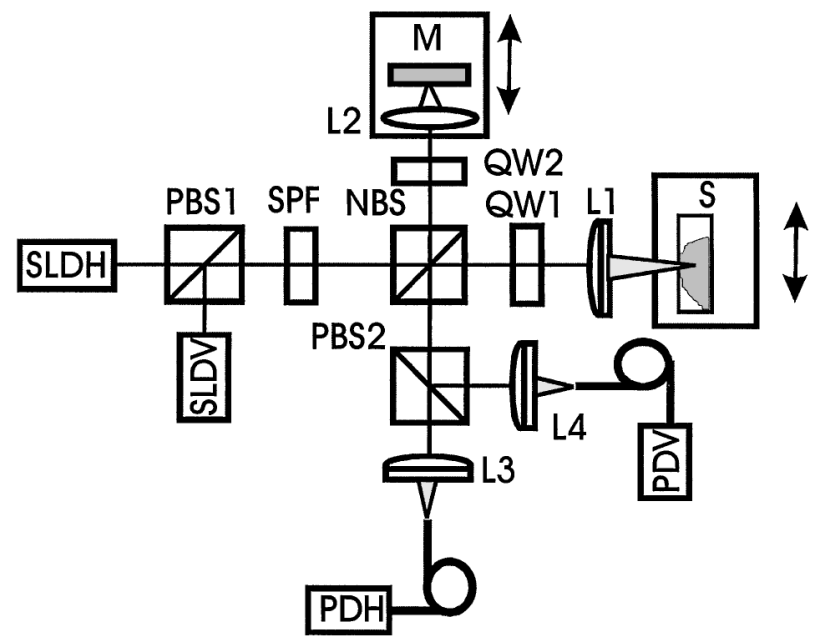

Fig. 1. Schematic of the double-beam polarization-sensitive OCT system: SLDH and SLDV are superluminescent diodes that are horizontally polarized $(\mathrm{H})$ and vertically polarized (V), respectively; PBS1, PBS2, polarizing beam splitters; SPF, spatial filter assembly; NBS, nonpolarizing beam splitter; QW1, QW2, zero-order quarter-wave plates; M, Mirror; L1-L4, lenses; S, sample. $\mathrm{PDH}$ and PDV are photodiodes for $\mathrm{H}$ and $\mathrm{V}$ polarization components, respectively. 
$\left(\lambda / 4\right.$ plate) oriented at $45^{\circ}$ and is focused into the sample by an objective lens $(\mathrm{L} 1 ; f=15 \mathrm{~mm}$, N.A. 0.15). The reference arm consists of a $\lambda / 4$ oriented at $22.5^{\circ}$, a lens (L2), and a mirror. After double passing through the $\lambda / 4$ plate, the horizontal polarization $(\mathrm{H})$ of the incident light is converted into $45^{\circ}$ polarization, and the vertical polarization $(\mathrm{V})$ of the incident light is converted into $-45^{\circ}$ polarization. The reference beam combines with the backscattered sample beam through the nonpolarizing beam splitter. The combined light is split into two orthogonal polarization components by polarizing beam splitter PBS2. The two components are coupled into two single-mode optical fibers, which are connected to photodiodes $\mathrm{PDH}$ and PDV, respectively. A data-acquisition board sampling at 50,000/s digitizes the two signals. A Doppler frequency of $\sim 1.2 \mathrm{kHz}$ is generated by the linear scan of the reference mirror. The carrier frequencies are the beat and harmonic frequencies between this Doppler frequency and the modulation frequencies of the light sources.

The incident Jones vector, $\mathbf{E}_{i}$, to the sample arm is transformed into the detected Jones vector, $\mathbf{E}_{0}$, as follows:

$$
\begin{aligned}
\mathbf{E}_{0} & =\mathbf{J}_{\mathrm{NBS}} \mathbf{J}_{\mathrm{QB}} \mathbf{J}_{\mathrm{SB}} \mathbf{J}_{\mathrm{M}} \mathbf{J}_{\mathrm{SI}} \mathbf{J}_{\mathrm{QI}} \mathbf{E}_{i} \\
& =\mathbf{J}_{\mathrm{NBS}} \mathbf{J}_{\mathrm{QB}} \mathbf{J} \mathbf{J}_{\mathrm{QI}} \mathbf{E}_{i}=\mathbf{J}_{T} \mathbf{E}_{i},
\end{aligned}
$$

where $\mathbf{J}_{\mathrm{QI}}$ and $\mathbf{J}_{\mathrm{QB}}$ are the Jones matrices of the $\lambda / 4$ plate for the incident and the backscattered light, respectively; $\mathbf{J}_{\mathrm{SI}}$ and $\mathbf{J}_{\mathrm{SB}}$ are the Jones matrices of the sample for the incident and the backscattered light, respectively; $\mathbf{J}_{M}$ is the Jones matrix of single backscattering - the same as the one for a mirror; $\mathbf{J}_{\mathrm{NBS}}$ is the Jones matrix of the reflecting surface of the nonpolarizing beam splitter; $\mathbf{J}$ is the combined Jones matrix of the scattering media; and $\mathbf{J}_{T}$ is the overall Jones matrix. As a consequence of the reciprocal constraint, ${ }^{7}$ the matrices $\mathbf{J}$ and $\mathbf{J}_{T}$ are transpose symmetric if the OCT signal is primarily from single backscattering and the contribution from multiple scattering can be neglected.

We bandpass filter the interference signals with central frequencies of 4.2 and $4.7 \mathrm{kHz}$ and a bandwidth of $10 \mathrm{~Hz}$ - the harmonic frequencies of the interference signals of sources $\mathrm{H}$ and $\mathrm{V}$, respectively- to extract the interference components of each light source. The two orthogonal interference components ( $\mathrm{H}$ and V) form the imaginary parts of the output Jones vectors, whose real parts are obtained through inverse Hilbert transformation. ${ }^{8}$ When the output Jones vectors for the two independent incident polarization states are determined, the elements of the Jones matrix can then be calculated based on Eq. (2).

The system was first tested by measurement of the matrix of a standard sample, a $\lambda / 4$ plate at various orientations in combination with a mirror. Figure 2(a) shows the amplitude of the vertical components of the measured Jones vector versus the orientation of the wave plate, where the amplitude of each Jones vector was normalized to unity. Figure 2(b) shows the phase differences between the vertical and the horizontal components of the Jones vectors. The calculated results were averaged over 1000 points centered at the peak of the interference signals, where 1000 points correspond to $10 \mu \mathrm{m}$, the resolution of the system. The results show that the measured data agree very well with the theoretical values.

The system was then applied to image soft tissue (a piece of porcine tendon). The tendon was mounted in a cuvette filled with saline solution. The sample was transversely scanned with a step size of $5 \mu \mathrm{m}$, and multiple A-scan images were taken. We first bandpass filtered the digitized interference signals with software and Hilbert transformed them to extract the analytical signal of each polarization component. For each A scan, the pixels were formed by averaging of the calculated elements of the Jones matrix over segments of 1000 points. Two-dimensional (2D) images were formed from these A-scan images and then median filtered. The final $2 \mathrm{D}$ images are shown in Fig. 3.

Clear band structures can be seen in some of the images, especially in $M_{13}, M_{22}, M_{23}, M_{31}, M_{32}$, and $M_{33}$. The period of the band structure is $\sim 0.13 \mathrm{~mm}$. There is no such band structure present in the $M_{00}$ image, which is the image based on the intensity of the backscattered light. In other words, the $M_{00}$ image is free of the effect of polarization. We believe that the band structure is generated by the birefringence of the collagen fibers in the porcine tendon. The band structure is distributed quite uniformly in the measured region; therefore, the birefringence is also uniform in the measured area.

Although all the polarization properties of a sample are contained in the Mueller matrix implicitly, explicit polarization parameters can be extracted from the

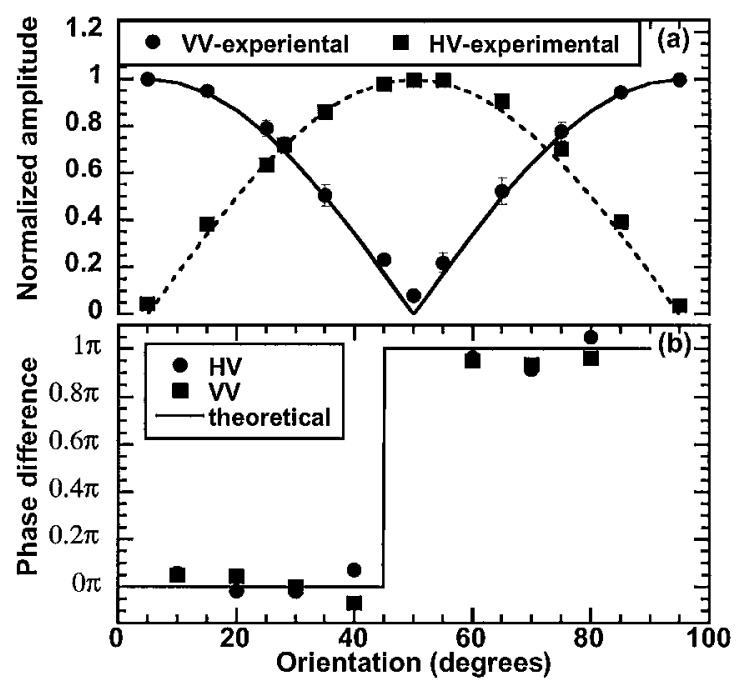

Fig. 2. (a) Normalized amplitude of the vertical components of the measured Jones vectors of a quarter-wave plate versus the orientation of the fast axis. HV is the horizontally polarized incident light, and VV is the vertically polarized incident light. The curves represent the expected theoretical values. (b) Phase differences between the vertical and the horizontal components of the Jones vectors of the same $\lambda / 4$ plate. The standard deviations are smaller than the symbols. 


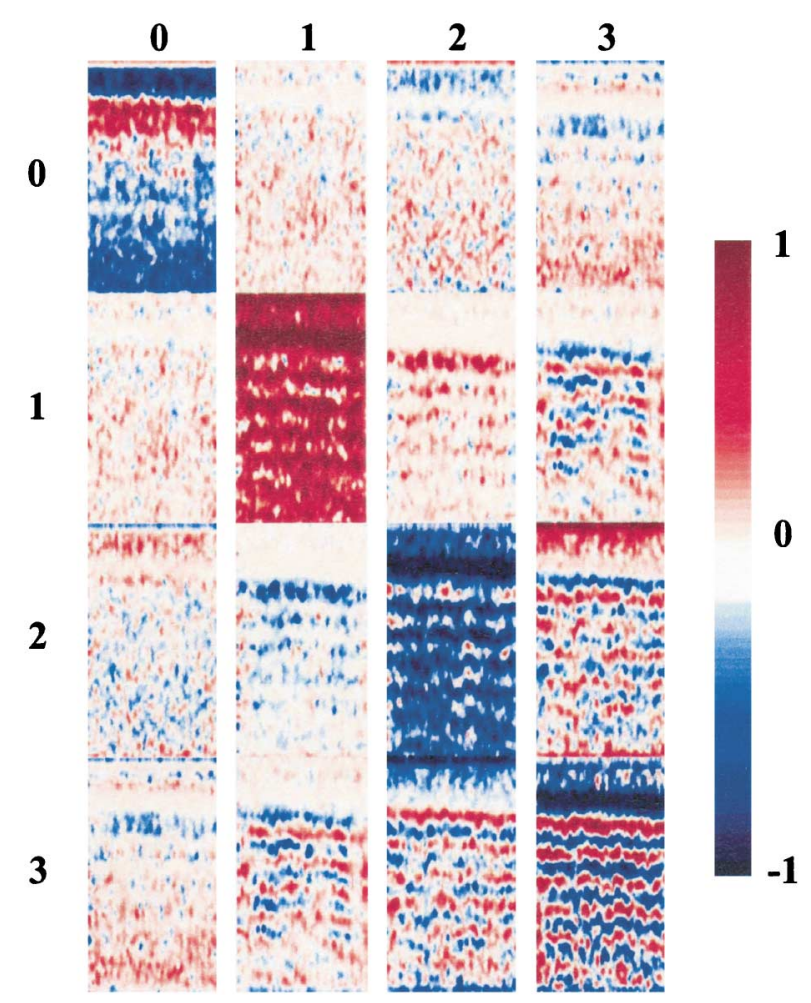

Fig. 3. 2D Mueller-matrix (M) images of a piece of porcine tendon. Each image except $M_{00}$ is pixelwise normalized with the $M_{00}$ element and shares the same color table. The size of each image is $0.5 \mathrm{~mm} \times 1 \mathrm{~mm}$.

matrix. The nondepolarizing Mueller matrix $\mathbf{M}$ can be decomposed by polar decomposition':

$$
\mathbf{M}=\mathbf{M}_{P} \mathbf{M}_{R},
$$

where $\mathbf{M}_{P}$ and $\mathbf{M}_{R}$ are the Mueller matrices of a diattenuator and an elliptical retarder, respectively. In biological tissues, it is reasonable to believe that the orientations of the diattenuator and retarder are the same. In this case, $\mathbf{M}$ is homogeneous in the polarization sense and the order of $\mathbf{M}_{P}$ and $\mathbf{M}_{R}$ in Eq. (3) is reversible. Because the effect of non-Faraday circular birefringence is canceled in the round-trip OCT signals, only linear birefringence exists in M. The magnitude of birefringence and the diattenuation are related to the density and property of collagen fibers in the sample, whereas the orientation of birefringence indicates the orientation of the collagen fibers.

We extracted polarization information from a piece of porcine tendon set at various orientations. The rotation axis of the sample was collinear with the optical axis (direction of incidence). The measurements were made at five different orientations with an interval of $10^{\circ}$. The $M_{31}$ and $M_{32}$ elements in Eq. (3) for a Mueller matrix that contains linear birefringence and linear or circular diattenuation can be expressed as

$$
\begin{aligned}
& M_{31}=P\left(P_{1}, P_{2}\right) \sin (2 \theta) \sin (\delta), \\
& M_{32}=-P\left(P_{1}, P_{2}\right) \cos (2 \theta) \sin (\delta),
\end{aligned}
$$

where $P$ is a function of the principal coefficients of the amplitude transmission, $P_{1}$ and $P_{2}$, for the two orthogo- nal polarization eigenstates of $\mathbf{M}_{P} ; \theta$ and $\delta$ are the orientation of the fast axis and the phase retardation of the retarder, respectively.

To increase the signal-to-noise ratio, we laterally averaged every 20 adjacent $\mathrm{A}$ scans of $M_{31}$ and $M_{32}$ in the calculated 2D images and fitted them for a physical depth of $0.4 \mathrm{~mm}$ (optical depth divided by the refractive index, which is assumed to be 1.4) from the surface. The calculated birefringence from the fitted data is $(4.2 \pm 0.3) \times 10^{-3}$, which is comparable with the previously reported value of $(3.7 \pm 0.4) \times 10^{-3}$ for bovine tendon. ${ }^{1}$ The calculated orientations of the fast axis are $(0 \pm 4)^{\circ},(9 \pm 2.9)^{\circ},(20.9 \pm 1.9)^{\circ},(30 \pm 2.8)^{\circ}$, and $(38 \pm 4.3)^{\circ}$ after subtraction of an offset angle. The small angular offset is due to the discrepancy between the actual and the observed fiber orientations. The results are very good considering that the tendon was slightly deformed when it was mounted in the cuvette and the rotation axis for the sample may not have been exactly collinear with the optical axis. The diattenuation, defined as $D=\left(P_{1}{ }^{2}-P_{2}{ }^{2}\right) /\left(P_{1}{ }^{2}+P_{2}{ }^{2}\right)=\left(M_{01}{ }^{2}+\right.$ $\left.M_{02}{ }^{2}+M_{03}{ }^{2}\right)^{1 / 2} / M_{00}$, was averaged over all the orientations and linearly fitted over a depth of $0.3 \mathrm{~mm}$. The fitted value of $D$ versus the round-trip physical path length increased with a slope of a $0.26 / \mathrm{mm}$ and reached $0.075 \pm 0.024$ at a depth of $0.3 \mathrm{~mm}$ after subtraction of an offset at the surface.

In summary, we have developed a novel double-beam polarization-sensitive OCT imaging technique. The Jones matrix of a sample can be determined with a single scan. This technique permits the acquisition of 2D tomographic Mueller-matrix images of either hard or soft biological tissues in vivo or in vitro. The Mueller matrix can be decomposed to yield important information on the optical polarization properties of a sample. The polarization properties can potentially be correlated with the conditions of biological tissues and thus used for disease diagnosis.

Thanks to G. Ku, Y. Xu, and D. Boas for helpful discussions. This project was sponsored in part by National Institutes of Health grants R21 RR15368 and R01 CA71980, by National Science Foundation grant BES-9734491, and by Texas Higher Education Coordinating Board grant 000512-0123-1999. L. V. Wang's e-mail address is LWang@tamu.edu.

\section{References}

1. J. F. de Boer, T. E. Milner, M. J. C. van Gemert, and J. S. Nelson, Opt. Lett. 22, 934 (1997).

2. M. J. Everett, K. Schoenerberger, B. W. Colston, Jr., and L. B. Da Silva, Opt. Lett. 23, 228 (1998).

3. J. F. de Boer, T. E. Milner, and J. S. Nelson, Opt. Lett. 24, 300 (1999).

4. G. Yao and L.-H. V. Wang, Opt. Lett. 24, 537 (1999).

5. S. Jiao, G. Yao, and L.-H. V. Wang, Appl. Opt. 39, 6318 (2000).

6. F. Le Roy-Brehonnet and B. Le Jeune, Prog. Quantum Electron. 21, 109 (1997).

7. N. Vansteenkiste, P. Vignolo, and A. Aspect, J. Opt. Soc. Am. A 10, 2240 (1993).

8. Y. Zhao, Z. Chen, C. Saxer, S. Xiang, J. F. de Boer, and J. S. Nelson, Opt. Lett. 25, 114 (2000).

9. J. J. Gil and E. Bernabeu, Optik 76, 67 (1987). 